\title{
Significant Excess of Electronlike Events in the MiniBooNE Short-Baseline Neutrino Experiment
}

A. A. Aguilar-Arevalo, ${ }^{13}$ B. C. Brown, ${ }^{6}$ L. Bugel, ${ }^{12}$ G. Cheng, ${ }^{5}$ J. M. Conrad,${ }^{12}$ R. L. Cooper,${ }^{10,15}$ R. Dharmapalan, ${ }^{1,2}$ A. Diaz, ${ }^{12}$ Z. Djurcic, ${ }^{2}$ D. A. Finley, ${ }^{6}$ R. Ford, ${ }^{6}$ F. G. Garcia,${ }^{6}$ G. T. Garvey,${ }^{10}$ J. Grange, ${ }^{7}$ E.-C. Huang, ${ }^{10}$ W. Huelsnitz, ${ }^{10}$ C. Ignarra, ${ }^{12}$ R. A. Johnson, ${ }^{3}$ G. Karagiorgi, ${ }^{5}$ T. Katori, ${ }^{1,16}$ T. Kobilarcik, ${ }^{6}$ W. C. Louis, ${ }^{10}$ C. Mariani, ${ }^{19}$ W. Marsh, ${ }^{6}$ G. B. Mills, ${ }^{10, *}$ J. Mirabal, ${ }^{10}$ J. Monroe, ${ }^{18}$ C. D. Moore, ${ }^{6}$ J. Mousseau, ${ }^{14}$ P. Nienaber, ${ }^{17}$ J. Nowak, ${ }^{9}$ B. Osmanov, ${ }^{7}$ Z. Pavlovic, ${ }^{6}$ D. Perevalov, ${ }^{6}$ H. Ray, ${ }^{7}$ B. P. Roe, ${ }^{14}$ A. D. Russell, ${ }^{6}$ M. H. Shaevitz, ${ }^{5}$ J. Spitz, ${ }^{14}$ I. Stancu, ${ }^{1}$ R. Tayloe, ${ }^{8}$ R. T. Thornton, ${ }^{10}$ M. Tzanov, ${ }^{4,11}$ R. G. Van de Water, ${ }^{10}$ D. H. White, ${ }^{10,}{ }^{*}$ D. A. Wickremasinghe, ${ }^{3}$ and E. D. Zimmerman ${ }^{4}$

(MiniBooNE Collaboration)

\author{
${ }^{1}$ University of Alabama, Tuscaloosa, Alabama 35487, USA \\ ${ }^{2}$ Argonne National Laboratory, Argonne, Illinois 60439, USA \\ ${ }^{3}$ University of Cincinnati, Cincinnati, Ohio 45221, USA \\ ${ }^{4}$ University of Colorado, Boulder, Colorado 80309, USA \\ ${ }^{5}$ Columbia University, New York, New York 10027, USA \\ ${ }^{6}$ Fermi National Accelerator Laboratory, Batavia, Illinois 60510, USA \\ ${ }^{7}$ University of Florida, Gainesville, Florida 32611, USA \\ ${ }^{8}$ Indiana University, Bloomington, Indiana 47405, USA \\ ${ }^{9}$ Lancaster University, Lancaster LA1 4YB, United Kingdom \\ ${ }^{10}$ Los Alamos National Laboratory, Los Alamos, New Mexico 87545, USA \\ ${ }^{11}$ Louisiana State University, Baton Rouge, Louisiana 70803, USA \\ ${ }^{12}$ Massachusetts Institute of Technology, Cambridge, Massachusetts 02139, USA \\ ${ }^{13}$ Instituto de Ciencias Nucleares, Universidad Nacional Autónoma de México, CDMX 04510, Mexico \\ ${ }^{14}$ University of Michigan, Ann Arbor, Michigan 48109, USA \\ ${ }^{15}$ New Mexico State University, Las Cruces, New Mexico 88003, USA \\ ${ }^{16}$ Queen Mary University of London, London E1 4NS, United Kingdom \\ ${ }^{17}$ Saint Mary's University of Minnesota, Winona, Minnesota 55987, USA \\ ${ }^{18}$ Royal Holloway, University of London, Egham TW20 OEX, United Kingdom \\ ${ }^{19}$ Center for Neutrino Physics, Virginia Tech, Blacksburg, Virginia 24061, USA
}

(Received 30 May 2018; revised manuscript received 28 September 2018; published 26 November 2018)

The MiniBooNE experiment at Fermilab reports results from an analysis of $\nu_{e}$ appearance data from $12.84 \times 10^{20}$ protons on target in neutrino mode, an increase of approximately a factor of 2 over previously reported results. A $\nu_{e}$ charged-current quasielastic event excess of $381.2 \pm 85.2$ events $(4.5 \sigma)$ is observed in the energy range $200<E_{\nu}^{\mathrm{QE}}<1250 \mathrm{MeV}$. Combining these data with the $\bar{\nu}_{e}$ appearance data from $11.27 \times 10^{20}$ protons on target in antineutrino mode, a total $\nu_{e}$ plus $\bar{\nu}_{e}$ charged-current quasielastic event excess of $460.5 \pm 99.0$ events $(4.7 \sigma)$ is observed. If interpreted in a two-neutrino oscillation model, $\nu_{\mu} \rightarrow \nu_{e}$, the best oscillation fit to the excess has a probability of $21.1 \%$, while the background-only fit has a $\chi^{2}$ probability of $6 \times 10^{-7}$ relative to the best fit. The MiniBooNE data are consistent in energy and magnitude with the excess of events reported by the Liquid Scintillator Neutrino Detector (LSND), and the significance of the combined LSND and MiniBooNE excesses is 6.0 $\sigma$. A two-neutrino oscillation interpretation of the data would require at least four neutrino types and indicate physics beyond the three neutrino paradigm. Although the data are fit with a two-neutrino oscillation model, other models may provide better fits to the data.

DOI: 10.1103/PhysRevLett.121.221801

\footnotetext{
Published by the American Physical Society under the terms of the Creative Commons Attribution 4.0 International license. Further distribution of this work must maintain attribution to the author(s) and the published article's title, journal citation, and DOI. Funded by $\operatorname{SCOAP}$.
} 
Evidence for short-baseline neutrino anomalies at an $L / E_{\nu} \sim 1 \mathrm{~m} / \mathrm{MeV}$, where $E_{\nu}$ is the neutrino energy and $L$ is the distance that the neutrino traveled before detection, comes from both neutrino appearance and disappearance experiments. The appearance anomalies include the excess of $\nu_{e}$ and $\bar{\nu}_{e}$ charge-current quasielastic (CCQE) events observed by the Liquid Scintillator Neutrino Detector (LSND) [1] and MiniBooNE [2,3] experiments, while the disappearance anomalies, although not completely consistent, include the deficit of $\nu_{e}$ and $\bar{\nu}_{e}$ events observed by reactor [4] and radioactive-source experiments [5]. As the masses and mixings within the three-generation neutrino matrix have been attached to solar and long-baseline neutrino experiments, more exotic models are typically used to explain these anomalies, including, for example, $3+N$ neutrino oscillation models involving three active neutrinos and $N$ additional sterile neutrinos [6-14], resonant neutrino oscillations [15], Lorentz violation [16], sterile neutrino decay [17], sterile neutrino nonstandard interactions [18], and sterile neutrino extra dimensions [19]. This Letter presents improved MiniBooNE $\nu_{e}$ and $\bar{\nu}_{e}$ appearance results, assuming two-neutrino oscillations with probability $P=\sin ^{2}(2 \theta) \sin ^{2}\left(1.27 \Delta m^{2} L / E\right)$, where $\theta$ is the mixing angle, $\Delta m^{2}\left(\mathrm{eV}^{2} / c^{4}\right)$ is the difference in neutrino mass eigenstates squared, $L(\mathrm{~m})$ is the distance traveled by the neutrino, and $E(\mathrm{MeV})$ is the neutrino energy.

The booster neutrino beam (BNB) at Fermilab delivers to the MiniBooNE experiment a flux of neutrinos and antineutrinos that is simulated using information from external measurements [20]. The BNB is produced by $8 \mathrm{GeV}$ protons from the Fermilab booster interacting on a beryllium target inside a magnetic focusing horn. Depending on the polarity of the horn, either $\pi^{+}$are focused and $\pi^{-}$are defocused to produce a fairly pure beam of $\nu_{\mu}$, or $\pi^{-}$are focused and $\pi^{+}$are defocused to produce a somewhat pure beam of $\bar{\nu}_{\mu}$. In neutrino mode, the $\nu_{\mu}, \bar{\nu}_{\mu}, \nu_{e}$, and $\bar{\nu}_{e}$ flux contributions at the detector are $93.5 \%, 5.9 \%, 0.5 \%$, and $0.1 \%$, respectively, while in antineutrino mode, the flux contributions are $15.7 \%, 83.7 \%, 0.2 \%$, and $0.4 \%$, respectively. The $\nu_{\mu}$ and $\bar{\nu}_{\mu}$ fluxes peak at approximately 600 and $400 \mathrm{MeV}$, respectively.

The MiniBooNE detector is described in detail in Ref. [21]. The detector consists of a $12.2 \mathrm{~m}$ diameter sphere filled with 818 tonnes of pure mineral oil $\left(\mathrm{CH}_{2}\right)$ and is located $541 \mathrm{~m}$ from the beryllium target. The detector is covered by 1520 8-inch photomultiplier tubes (PMTs), where 1280 PMTs are in the interior detector region and 240 PMTs are located in the optically isolated outer veto region. Charged particles produced by neutrino interactions in the mineral oil emit both directed Cherenkov light and isotropic scintillation light that is detected by the PMTs. Event reconstruction [22] and particle identification make use of the hit PMT charge and time information, and the reconstructed neutrino energy $E_{\nu}^{\mathrm{QE}}$ is estimated from the measured energy and angle of the outgoing muon or electron, assuming the kinematics of CCQE scattering [23].

From 2002-2017, the MiniBooNE experiment has collected a total of $11.27 \times 10^{20}$ protons on target (POT) in antineutrino mode, $12.84 \times 10^{20}$ POT in neutrino mode, and a further $1.86 \times 10^{20} \mathrm{POT}$ in a special beam-off target mode to search for sub-GeV dark matter [24]. The neutrino sample has approximately doubled in size since the previous publication [3]. The published neutrino-mode data correspond to $6.46 \times 10^{20}$ POT, while $6.38 \times 10^{20}$ POT were obtained in 2016 and 2017. During the 15 years of running, the BNB and MiniBooNE detector have been stable to within $2 \%$ in neutrino energy.

The analysis is optimized to measure $\nu_{e}$ and $\bar{\nu}_{e}$ induced CCQE events, and the event reconstruction [22] and selection are identical to the previous analysis [3]. The average selection efficiency is $\sim 20 \%(\sim 0.1 \%)$ for $\nu_{e}-$ induced CCQE events $\left(\nu_{\mu}\right.$-induced background events) generated over the fiducial volume. The fraction of CCQE events in antineutrino mode that are from wrongsign neutrino events was determined from the angular distributions of muons created in CCQE interactions and by measuring CC single $\pi^{+}$events [25].

The predicted but unconstrained $\nu_{e}$ and $\bar{\nu}_{e}$ CCQE background events for the neutrino energy range $200<$ $E_{\nu}^{\mathrm{QE}}<1250 \mathrm{MeV}$ are shown in Table I for both neutrino

TABLE I. The expected (unconstrained) number of events for the $200<E_{\nu}^{\mathrm{QE}}<1250 \mathrm{MeV}$ neutrino energy range from all of the backgrounds in the $\nu_{e}$ and $\bar{\nu}_{e}$ appearance analysis before using the constraint from the $\mathrm{CC} \nu_{\mu}$ events. Also shown are the constrained background, as well as the expected number of events corresponding to the LSND best fit oscillation probability of $0.26 \%$, assuming oscillations at large $\Delta m^{2}$. The table shows the diagonal-element systematic plus statistical uncertainties, which become substantially reduced in the oscillation fits when correlations between energy bins and between the electron and muon neutrino events are included. The antineutrino numbers are from a previous analysis [3].

\begin{tabular}{lcc}
\hline \hline Process & Neutrino mode & Antineutrino mode \\
\hline$\nu_{\mu} \& \bar{\nu}_{\mu}$ CCQE & $73.7 \pm 19.3$ & $12.9 \pm 4.3$ \\
NC $\pi^{0}$ & $501.5 \pm 65.4$ & $112.3 \pm 11.5$ \\
NC $\Delta \rightarrow N \gamma$ & $172.5 \pm 24.1$ & $34.7 \pm 5.4$ \\
External events & $75.2 \pm 10.9$ & $15.3 \pm 2.8$ \\
Other $\nu_{\mu} \& \bar{\nu}_{\mu}$ & $89.6 \pm 22.9$ & $22.3 \pm 3.5$ \\
$\nu_{e} \& \bar{\nu}_{e}$ from $\mu^{ \pm}$decay & $425.3 \pm 100.2$ & $91.4 \pm 27.6$ \\
$\nu_{e} \& \bar{\nu}_{e}$ from $K^{ \pm}$decay & $192.2 \pm 41.9$ & $51.2 \pm 11.0$ \\
$\nu_{e} \& \bar{\nu}_{e}$ from $K_{L}^{0}$ decay & $54.5 \pm 20.5$ & $51.4 \pm 18.0$ \\
Other $\nu_{e} \& \bar{\nu}_{e}$ & $6.0 \pm 3.2$ & $6.7 \pm 6.0$ \\
Unconstrained bkgd. & $1590.6 \pm 176.9$ & $398.2 \pm 49.7$ \\
Constrained bkgd. & $1577.8 \pm 85.2$ & $398.7 \pm 28.6$ \\
Total data & 1959 & 478 \\
Excess & $381.2 \pm 85.2$ & $79.3 \pm 28.6$ \\
$0.26 \%(\mathrm{LSND}) \nu_{\mu} \rightarrow \nu_{e}$ & 463.1 & 100.0 \\
\hline \hline
\end{tabular}


mode and antineutrino mode [26]. See Supplemental Material [27] for more information on backgrounds. The upper limit of $1250 \mathrm{MeV}$ corresponded to a small value of $L / E$ and was chosen by the collaboration before unblinding the data in 2007. The lower limit of $200 \mathrm{MeV}$ is chosen because we constrain the $\nu_{e}$ events with the CCQE $\nu_{\mu}$ events and our CCQE $\nu_{\mu}$ event sample only goes down to $200 \mathrm{MeV}$, as we require a visible Cherenkov ring from the muon. The estimated sizes of the intrinsic $\nu_{e}$ and gamma backgrounds are based on MiniBooNE event measurements and uncertainties from these constraints are included in the analysis. The intrinsic $\nu_{e} / \bar{\nu}_{e}$ background from muon decay is directly related to the large sample of observed $\nu_{\mu} / \bar{\nu}_{\mu}$ events, as these events constrain the muons that decay in the $50 \mathrm{~m}$ decay region. This constraint uses a joint fit of the observed $\nu_{\mu} / \bar{\nu}_{\mu}$ and $\nu_{e} / \bar{\nu}_{e}$ events, assuming that there are no substantial $\nu_{\mu} / \bar{\nu}_{\mu}$ disappearance oscillations. The other intrinsic $\nu_{e}$ background component, from kaon decay, is constrained by fits to kaon production data and SciBooNE measurements [28]. The intrinsic $\nu_{e}$ background from pion decay $\left(1.2 \times 10^{-4}\right.$ branching ratio $)$ and hyperon decay are very small. Other backgrounds from misidentified $\nu_{\mu}$ or $\bar{\nu}_{\mu}[29,30]$ events are also constrained by the observed CCQE sample.

The gamma background from neutral-current (NC) $\pi^{0}$ production and $\Delta \rightarrow N \gamma$ radiative decay [31,32] are constrained by the associated large two-gamma sample (mainly from $\Delta$ production) observed in the MiniBooNE data, where $\pi^{0}$ measurements [33] are used to constrain the $\pi^{0}$ background. The $\pi^{0}$ background measured in the first and second neutrino data sets were found to be consistent, resulting in a lower statistical background uncertainty for the combined data. Other neutrino-induced single gamma production processes are included in the theoretical predictions, which agree well with the MiniBooNE estimates [31,34]. Single-gamma backgrounds from external neutrino interactions ("dirt" backgrounds) are estimated using topological and spatial cuts to isolate the events whose vertices are near the edge of the detector and point towards the detector center [35]. With the larger data set, the background from external neutrino interactions is now better determined to be approximately $7 \%$ larger, but with smaller uncertainty than in the previous publication [3]. A new technique to measure or constrain the gamma and dirt backgrounds based on event timing relative to the beam is in development.

Systematic uncertainties are determined by considering the predicted effects on the $\nu_{\mu}, \bar{\nu}_{\mu}, \nu_{e}$, and $\bar{\nu}_{e}$ CCQE rates from variations of uncertainty parameters. The parameters include uncertainties in the neutrino and antineutrino flux estimates, uncertainties in neutrino cross sections, most of which are determined by in situ cross-section measurements at MiniBooNE [29,33], uncertainties from nuclear effects, and uncertainties in detector modeling and reconstruction. A covariance matrix in bins of $E_{\nu}^{\mathrm{QE}}$ is

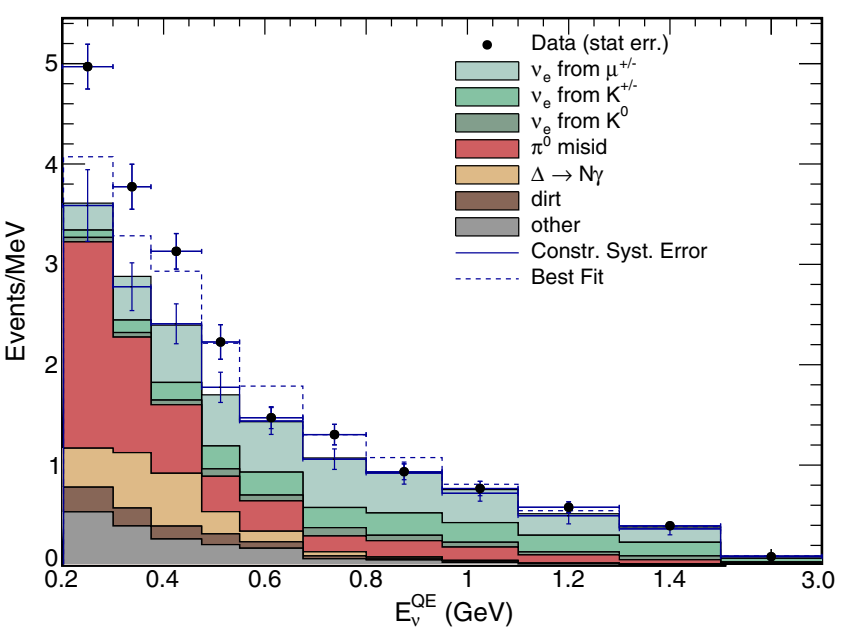

FIG. 1. The MiniBooNE neutrino mode $E_{\nu}^{\mathrm{QE}}$ distributions, corresponding to the total $12.84 \times 10^{20}$ POT data, for $\nu_{e} \mathrm{CCQE}$ data (points with statistical errors) and background (histogram with systematic errors). The dashed curve shows the best fit to the neutrino-mode data assuming two-neutrino oscillations. The last bin is for the energy interval from $1500-3000 \mathrm{MeV}$.

constructed by considering the variation from each source of systematic uncertainty on the $\nu_{e}$ and $\bar{\nu}_{e} \mathrm{CCQE}$ signal and background, and the $\nu_{\mu}$ and $\bar{\nu}_{\mu}$ CCQE prediction as a function of $E_{\nu}^{\mathrm{QE}}$. This matrix includes correlations between any of the $\nu_{e}$ and $\bar{\nu}_{e}$ CCQE signal and background and $\nu_{\mu}$ and $\bar{\nu}_{\mu} \mathrm{CCQE}$ samples, and is used in the $\chi^{2}$ calculation of the oscillation fits.

Table I also shows the expected number of events corresponding to the LSND best fit oscillation probability of $0.26 \%$, assuming oscillations at large $\Delta m^{2}$. LSND and MiniBooNE have the same average value of $L / E$, but MiniBooNE has a larger range of $L / E$. Therefore, the appearance probabilities for LSND and MiniBooNE should not be exactly the same at lower $L / E$ values.

Figure 1 shows the $E_{\nu}^{\mathrm{QE}}$ distribution for $\nu_{e} \mathrm{CCQE}$ data and background in neutrino mode for the total $12.84 \times 10^{20} \mathrm{POT}$ data. Each bin of reconstructed $E_{\nu}^{\mathrm{QE}}$ corresponds to a distribution of "true" generated neutrino energies, which can overlap adjacent bins. In neutrino mode, a total of 1959 data events pass the $\nu_{e} \mathrm{CCQE}$ event selection requirements with $200<E_{\nu}^{\mathrm{QE}}<1250 \mathrm{MeV}$, compared to a background expectation of $1577.8 \pm 39.7$ (stat) \pm 75.4 (syst) events. The excess is then $381.2 \pm 85.2$ events or a $4.5 \sigma$ effect. Note that the 162.0 event excess in the first $6.46 \times 10^{20}$ POT data is approximately $1 \sigma$ lower than the average excess, while the 219.2 event excess in the second $6.38 \times 10^{20}$ POT data is approximately $1 \sigma$ higher than the average excess. Figure 2 shows the excess events in neutrino mode from the first $6.46 \times 10^{20}$ POT data and the second $6.38 \times 10^{20}$ POT data (top plot). Combining the MiniBooNE neutrino and antineutrino data, there are a total of 2437 events in the $200<$ $E_{\nu}^{\mathrm{QE}}<1250 \mathrm{MeV}$ energy region, compared to a background 


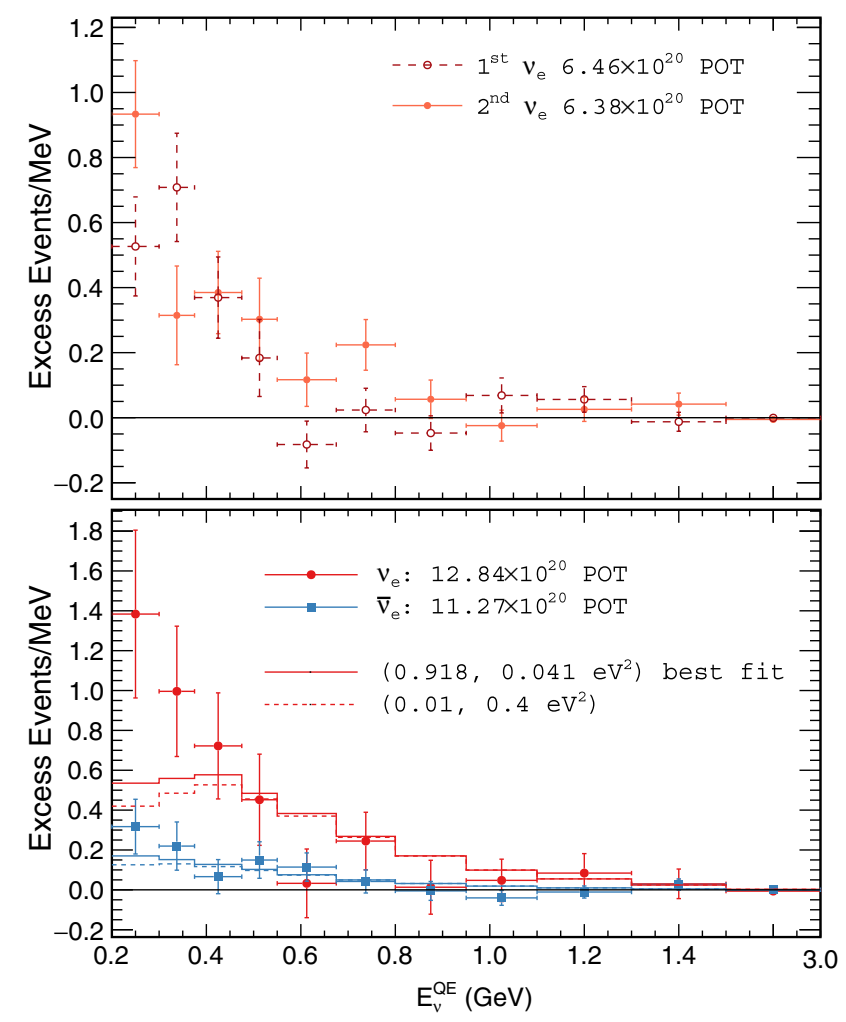

FIG. 2. The top plot shows the MiniBooNE event excesses in neutrino mode as a function of $E_{\nu}^{\mathrm{QE}}$ from the first $6.46 \times 10^{20}$ POT data and the second $6.38 \times 10^{20}$ POT data. The bottom plot shows the total event excesses in both neutrino mode and antineutrino mode, corresponding to $12.84 \times 10^{20}$ POT and $11.27 \times 10^{20}$ POT, respectively. The solid (dashed) curve is the best fit ( $1 \sigma$ fit point) to the neutrino-mode and antineutrino-mode data assuming two-neutrino oscillations. The last bin is for the energy interval from 1500-3000 MeV. Error bars include only statistical uncertainties for the top plot and both statistical and correlated systematic uncertainties for the bottom plot.

expectation of $1976.5 \pm 44.5$ (stat) \pm 88.5 (syst) events. This corresponds to a total $\nu_{e}$ plus $\bar{\nu}_{e}$ CCQE excess of $460.5 \pm 99.0$ events with respect to expectation or a $4.7 \sigma$ excess. Figure 2 (bottom plot) shows the total event excesses as a function of $E_{\nu}^{\mathrm{QE}}$ in both neutrino mode and antineutrino mode. The dashed curves show the two-neutrino oscillation predictions at the best-fit point $\left(\Delta m^{2}=0.041 \mathrm{eV}^{2}\right.$, $\sin ^{2} 2 \theta=0.92$ ), as well as at a point within $1 \sigma$ of the best-fit point $\left(\Delta m^{2}=0.4 \mathrm{eV}^{2}, \sin ^{2} 2 \theta=0.01\right)$.

A two-neutrino model is assumed for the MiniBooNE oscillation fits in order to compare with the LSND data. However, the appearance neutrino experiments appear to be incompatible with the disappearance neutrino experiments in a $3+1$ model $[10,12]$, and other models [15-19] may provide better fits to the data. The oscillation parameters are extracted from a combined fit of the observed $E_{\nu}^{\mathrm{QE}}$ event distributions for muonlike and electronlike events using the full covariance matrix described previously in the full

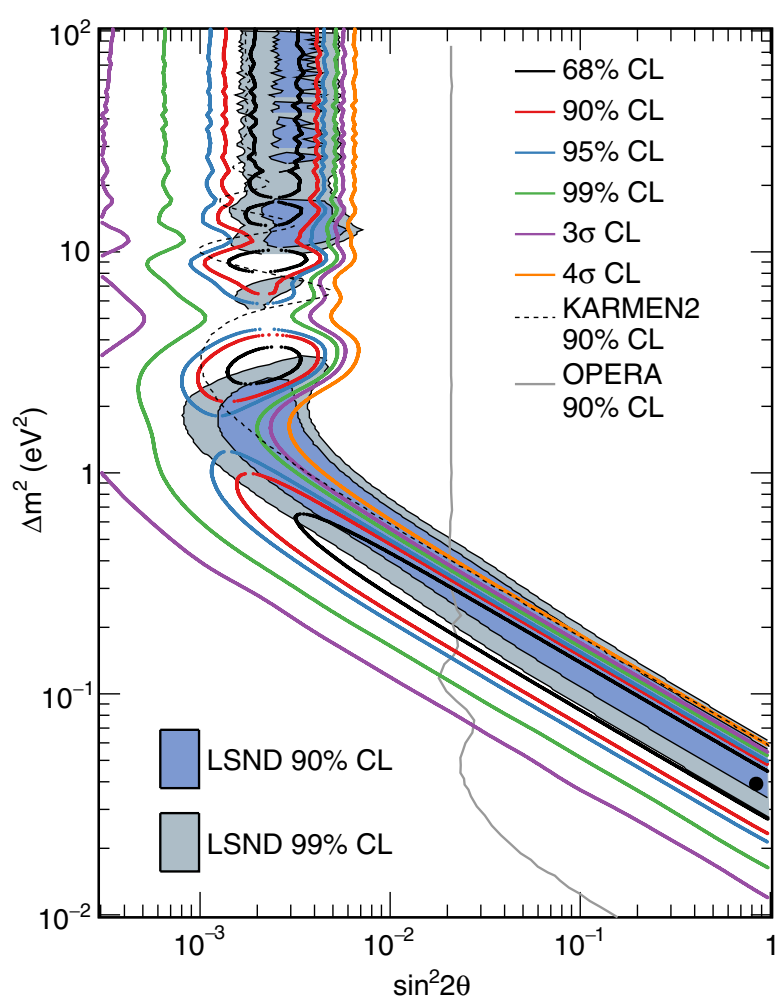

FIG. 3. MiniBooNE allowed regions in neutrino mode $\left(12.84 \times 10^{20}\right.$ POT) for events with $200<E_{\nu}^{\mathrm{QE}}<3000 \mathrm{MeV}$ within a two-neutrino oscillation model. The shaded areas show the $90 \%$ and $99 \%$ C.L. LSND $\bar{\nu}_{\mu} \rightarrow \bar{\nu}_{e}$ allowed regions. The black point shows the MiniBooNE best fit point. Also shown are 90\% C.L. limits from the KARMEN [36] and OPERA [37] experiments.

energy range $200<E_{\nu}^{\mathrm{QE}}<3000 \mathrm{MeV}$. The fit assumes the same oscillation probability for both the right-sign $\nu_{e}$ and wrong-sign $\bar{\nu}_{e}$, and no $\nu_{\mu}, \bar{\nu}_{\mu}, \nu_{e}$, or $\bar{\nu}_{e}$ disappearance. Using a likelihood-ratio technique [3], the confidence level values for the fitting statistic, $\Delta \chi^{2}=\chi^{2}$ (point) $-\chi^{2}$ (best), as a function of oscillation parameters, $\Delta m^{2}$ and $\sin ^{2} 2 \theta$, is determined from frequentist, fake data studies. The fake data studies also determine the effective number of degrees of freedom and probabilities. With this technique, the best neutrino oscillation fit in neutrino mode occurs at $\left(\Delta m^{2}, \sin ^{2} 2 \theta\right)=\left(0.039 \mathrm{eV}^{2}, 0.84\right)$, as shown in Fig. 3. The $\chi^{2} / n d f$ for the best-fit point in the energy range $200<$ $E_{\nu}^{\mathrm{QE}}<1250 \mathrm{MeV}$ is $9.9 / 6.7$ with a probability of $15.5 \%$. The background-only fit has a $\chi^{2}$ probability of $0.06 \%$ relative to the best oscillation fit and a $\chi^{2} / n d f=24.9 / 8.7$ with a probability of $0.21 \%$. Figure 3 shows the MiniBooNE closed confidence level (C.L.) contours for $\nu_{e}$ appearance oscillations in neutrino mode in the $200<E_{\nu}^{\mathrm{QE}}<3000 \mathrm{MeV}$ energy range.

Nuclear effects associated with neutrino interactions on carbon can affect the reconstruction of the neutrino energy, $E_{\nu}^{\mathrm{QE}}$, and the determination of the neutrino oscillation 


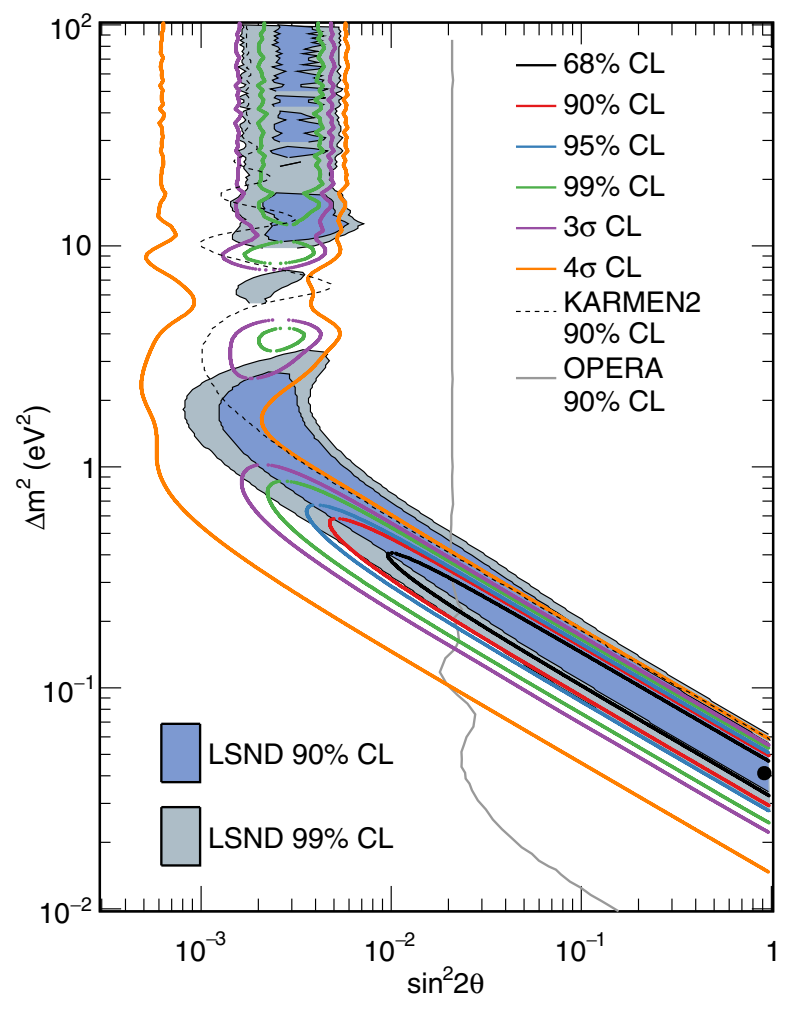

FIG. 4. MiniBooNE allowed regions for a combined neutrino mode $\left(12.84 \times 10^{20}\right.$ POT $)$ and antineutrino mode $\left(11.27 \times 10^{20}\right.$ POT) data sets for events with $200<E_{\nu}^{\mathrm{QE}}<3000 \mathrm{MeV}$ within a two-neutrino oscillation model. The shaded areas show the $90 \%$ and $99 \%$ C.L. LSND $\bar{\nu}_{\mu} \rightarrow \bar{\nu}_{e}$ allowed regions. The black point shows the MiniBooNE best fit point. Also shown are $90 \%$ C.L. limits from the KARMEN [36] and OPERA [37] experiments.

parameters [38]. These effects were studied previously $[3,39]$ and were found to not affect substantially the oscillation fit. In addition, they do not affect the gamma background, which is determined from direct measurements of $\mathrm{NC} \pi^{0}$ and dirt backgrounds.

Figure 4 shows the MiniBooNE allowed regions in both neutrino mode and antineutrino mode [3] for events with $200<E_{\nu}^{\mathrm{QE}}<3000 \mathrm{MeV}$ within a two-neutrino oscillation model. For this oscillation fit the entire data set is used and includes the $12.84 \times 10^{20}$ POT data in neutrino mode and the $11.27 \times 10^{20}$ POT data in antineutrino mode. As shown in the figure, the MiniBooNE $1 \sigma$ allowed region lies mostly within the LSND 90\% C.L. band, which demonstrates good agreement between the LSND and MiniBooNE signals. Also shown are 90\% C.L. limits from the KARMEN [36] and OPERA [37] experiments. The KARMEN2 90\% C.L. limits are outside the MiniBooNE 95\% C.L. allowed region, while the OPERA $90 \%$ C.L. limits disfavor the MiniBooNE allowed region below approximately $0.3 \mathrm{eV}^{2}$. The best combined neutrino oscillation fit occurs at $\left(\Delta m^{2}, \sin ^{2} 2 \theta\right)=\left(0.041 \mathrm{eV}^{2}, 0.92\right)$. The $\chi^{2} / n d f$ for the best-fit point in the energy range $200<E_{\nu}^{\mathrm{QE}}<1250 \mathrm{MeV}$

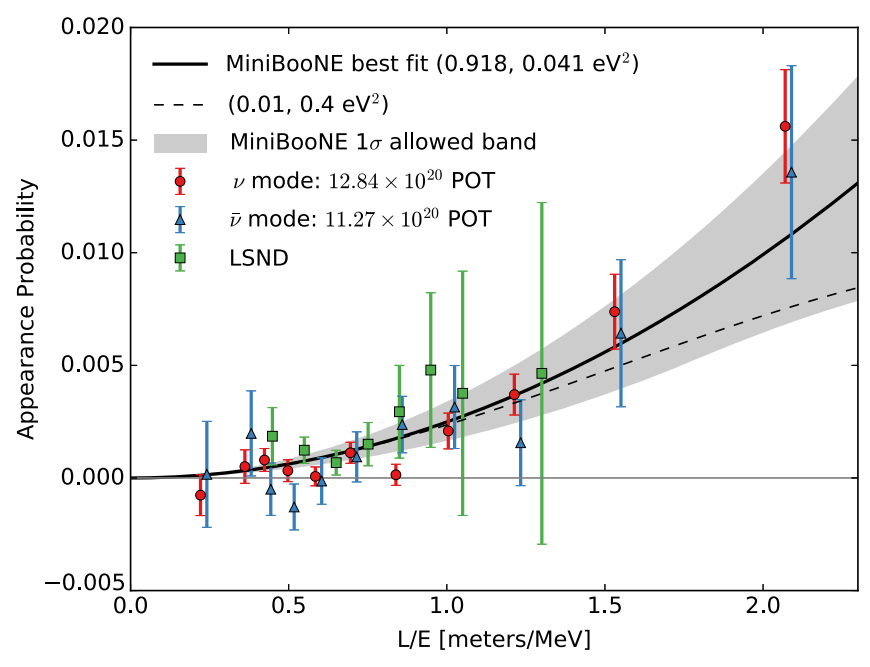

FIG. 5. A comparison between the $L / E_{\nu}^{\mathrm{QE}}$ distributions for the MiniBooNE data excesses in neutrino mode $\left(12.84 \times 10^{20}\right.$ POT $)$ and antineutrino mode $\left(11.27 \times 10^{20}\right.$ POT $)$ to the $L / E$ distribution from LSND [1]. The error bars show statistical uncertainties only. The curves show fits to the MiniBooNE data, assuming twoneutrino oscillations, while the shaded area is the MiniBooNE $1 \sigma$ allowed band. The best-fit curve uses the reconstructed neutrino energy $E_{\nu}^{\mathrm{QE}}$ for the MiniBooNE data. The dashed curve shows the example $1 \sigma$ fit point.

is $19.4 / 15.6$ with a probability of $21.1 \%$, and the background-only fit has a $\chi^{2}$ probability of $6 \times 10^{-7}$ relative to the best oscillation fit and a $\chi^{2} / n d f=47.1 / 17.3$ with a probability of $0.02 \%$.

Figure 5 compares the $L / E_{\nu}^{\mathrm{QE}}$ distributions for the MiniBooNE data excesses in neutrino mode and antineutrino mode to the $L / E$ distribution from LSND [1]. The error bars show statistical uncertainties only. As shown in the figure, there is agreement among all three data sets. Assuming two-neutrino oscillations, the curves show fits to the MiniBooNE data described above. Fitting both MiniBooNE and LSND data, by adding LSND $L / E$ data as additional terms, the best fit occurs at $\left(\Delta m^{2}, \sin ^{2} 2 \theta\right)=$ $\left(0.041 \mathrm{eV}^{2}, 0.96\right)$ with a $\chi^{2} / n d f=22.4 / 22.4$, corresponding to a probability of $42.5 \%$. The MiniBooNE excess of events in both oscillation probability and $L / E$ spectrum is, therefore, consistent with the LSND excess of events. The significance of the combined LSND (3.8 $\sigma)$ [1] and MiniBooNE (4.7 $\sigma$ ) excesses is $6.0 \sigma$, which is obtained by adding the significances in quadrature, as the two experiments have completely different neutrino energies, neutrino fluxes, reconstructions, backgrounds, and systematic uncertainties.

In summary, the MiniBooNE experiment observes a total $\nu_{e}$ CCQE event excess in both neutrino and antineutrino running modes of $460.5 \pm 99.0$ events $(4.7 \sigma)$ in the energy range $200<E_{\nu}^{\mathrm{QE}}<1250 \mathrm{MeV}$. The MiniBooNE allowed region from a two-neutrino oscillation fit to the data, shown in Fig. 4, is consistent with the allowed region reported by 
the LSND experiment [1]. On the other hand, a twoneutrino oscillation interpretation of the data would require at least four neutrino types and indicate physics beyond the three neutrino paradigm. The significance of the combined LSND and MiniBooNE excesses is $6.0 \sigma$. All of the major backgrounds are constrained by in situ event measurements, so nonoscillation explanations would need to invoke new anomalous background processes. Although the data are fit with a two-neutrino oscillation model, other models may provide better fits to the data. The MiniBooNE event excess will be further studied by the Fermilab shortbaseline neutrino $(\mathrm{SBN})$ program [40].

We acknowledge the support of Fermilab, the Department of Energy, and the National Science Foundation, and we acknowledge Los Alamos National Laboratory for LDRD funding.

*Deceased.

[1] C. Athanassopoulos et al., Phys. Rev. Lett. 75, 2650 (1995); 77, 3082 (1996); 81, 1774 (1998); Phys. Rev. C. 54, 2685 (1996); 58, 2489 (1998); A. Aguilar et al., Phys. Rev. D 64, 112007 (2001).

[2] A. A. Aguilar-Arevalo et al., Phys. Rev. Lett. 98, 231801 (2007); 102, 101802 (2009); 105, 181801 (2010).

[3] A. A. Aguilar-Arevalo et al., Phys. Rev. Lett. 110, 161801 (2013).

[4] G. Mention, M. Fechner, T. Lasserre, T. A. Mueller, D. Lhuillier, M. Cribier, and A. Letourneau, Phys. Rev. D 83, 073006 (2011).

[5] C. Giunti and M. Laveder, Phys. Rev. C 83, 065504 (2011).

[6] M. Sorel, J. M. Conrad, and M. H. Shaevitz, Phys. Rev. D 70, 073004 (2004).

[7] G. Karagiorgi, Z. Djurcic, J. M. Conrad, M. H. Shaevitz, and M. Sorel, Phys. Rev. D 80, 073001 (2009); 81, 039902(E) (2010).

[8] G. H. Collin, C. A. Argüelles, J. M. Conrad, and M. H. Shaevitz, Phys. Rev. Lett. 117, 221801 (2016).

[9] C. Giunti and M. Laveder, Phys. Lett. B 706, 200 (2011); Phys. Rev. D 84, 073008 (2011).

[10] S. Gariazzo, C. Giunti, M. Laveder, and Y. F. Li, J. High Energy Phys. 06 (2017) 135.

[11] J. Kopp, M. Maltoni, and T. Schwetz, Phys. Rev. Lett. 107, 091801 (2011); J. Kopp and P. A. N. Machado, J. High Energy Phys. 05 (2013) 050.

[12] M. Dentler, A. Hernandez-Cabezudo, J. Kopp, P. Machado, M. Maltoni, I. Martinez-Soler, and T. Schwetz, J. High Energy Phys. 08 (2018) 010.

[13] K. N. Abazajian et al., arXiv:1204.5379.

[14] J. M. Conrad, C. M. Ignarra, G. Karagiorgi, M. H. Shaevitz, and J. Spitz, Adv. High Energy Phys. 2013, 163897 (2013).

[15] J. Asaadi, E. Church, R. Guenette, B. J. P. Jones, and A. M. Szelc, Phys. Rev. D 97, 075021 (2018); G. Karagiorgi, M. H. Shaevitz, and J. M. Conrad, arXiv:1202.1024; H. Pas, S. Pakvasa, and T. J. Weiler, Phys. Rev. D 72, 095017 (2005); D. Doring, H. Paes, P. Sicking, and T. J. Weiler, arXiv: 1808.07460 .
[16] V. A. Kostelecky and M. Mewes, Phys. Rev. D 69, 016005 (2004); T. Katori, V. A. Kostelecky, and R. Tayloe, Phys. Rev. D 74, 105009 (2006); J. S. Diaz and V. A. Kostelecky, Phys. Lett. B 700, 25 (2011); Phys. Rev. D 85, 016013 (2012).

[17] S. N. Gninenko, Phys. Rev. Lett. 103, 241802 (2009); S. N. Gninenko and D. S. Gorbunov, Phys. Rev. D 81, 075013 (2010); Y. Bai, R. Lu, S. Lu, J. Salvado, and B. A. Stefanek, Phys. Rev. D 93, 073004 (2016); Z. Moss, M. H. Moulai, C. A. Argüelles, and J. M. Conrad, Phys. Rev. D 97, 055017 (2018); E. Baertuzzo, S. Jana, P. A. N. Machado, and R. Z. Funchal, arXiv:1807.09877; P. Ballett, S. Pascoli, and M. Ross-Lonergan, arXiv:1808.02915.

[18] J. Liao and D. Marfatia, Phys. Rev. Lett. 117, 071802 (2016).

[19] M. Carena, Y.-Y. Li, C. S. Machado, and P. A. N. Machado, and C. E. M. Wagner, Phys. Rev. D 96, 095014 (2017).

[20] A. A. Aguilar-Arevalo et al., Phys. Rev. D 79, 072002 (2009).

[21] A. A. Aguilar-Arevalo et al., Nucl. Instrum. Methods Phys. Res., Sect. A 599, 28 (2009).

[22] R. B. Patterson, E. M. Laird, Y. Liu, P. D. Meyers, I. Stancu, and H. A. Tanaka, Nucl. Instrum. Methods Phys. Res., Sect. A 608, 206 (2009).

[23] A. A. Aguilar-Arevalo et al., Phys. Rev. D 81, 092005 (2010); Phys. Rev. D 88, 032001 (2013).

[24] A. A. Aguilar-Arevalo et al. (MiniBooNE Collaboration), Phys. Rev. Lett. 118, 221803 (2017); arXiv:1807.06137.

[25] A. A. Aguilar-Arevalo et al., Phys. Rev. D 84, 072005 (2011).

[26] R. B. Patterson, Ph. D. thesis, Princeton University, 2007, http://www-boone.fnal.gov/publications/Papers/rbpatter_ thesis.pdf.

[27] See Supplemental Material at http://link.aps.org/ supplemental/10.1103/PhysRevLett.121.221801 for more information on backgrounds and plots showing agreement between data and the Monte Carlo simulation.

[28] G. Cheng et al., Phys. Rev. D 84, 012009 (2011); C. Mariani, G. Cheng, J. M. Conrad, and M. H. Shaevitz, Phys. Rev. D 84, 114021 (2011).

[29] A. A. Aguilar-Arevalo et al., Phys. Rev. D 81, 092005 (2010); Phys. Rev. Lett. 100, 032301 (2008).

[30] A. A. Aguilar-Arevalo et al., Phys. Rev. D 83, 052007 (2011); Phys. Rev. Lett. 103, 081801 (2009).

[31] V. P. Efrosinin, Yu. G. Kudenko, and A. N. Khotjantsev, Phys. At. Nucl. 72, 459 (2009); R. J. Hill, Phys. Rev. D 81, 013008 (2010); , Phys. Rev. D 84, 017501 (2011); X. Zhang and B. D. Serot, Phys. Lett. B 719, 409 (2013); Phys. Rev. C 86, 035502 (2012); 86, 035504 (2012); B. D. Serot and X. Zhang, Phys. Rev. C 86, 015501 (2012).

[32] E. Wang, L. Alvarez-Ruso, and J. Nieves, Phys. Rev. C 89, 015503 (2014); Phys. Lett. B 740, 16 (2015).

[33] A. A. Aguilar-Arevalo et al., Phys. Rev. D 81, 013005 (2010); Phys. Lett. B 664, 41 (2008).

[34] D. Rein and L. M. Sehgal, Phys. Lett. 104B, 394 (1981); S. S. Gershtein, Yu. Ya. Komachenko, and M. Yu. Khlopov, Sov. J. Nucl. Phys. 33, 860 (1981); J. A. Harvey, C. T. Hill, and R. J. Hill, Phys. Rev. Lett. 99, 261601 (2007); J. P. Jenkins and T. Goldman, Phys. Rev. D 80, 053005 (2009); A. M. Ankowski, O. Benhar, T. Mori, R. Yamaguchi, and M. Sakuda, Phys. Rev. 
Lett. 108, 052505 (2012); K. M. Graczyk, D. Kielczewska, P. Przewlocki, and J. T. Sobczyk, Phys. Rev. D 80, 093001 (2009).

[35] A. A. Aguilar-Arevalo et al., Phys. Rev. Lett. 102, 101802 (2009).

[36] B. Armbruster et al., Phys. Rev. D 65, 112001 (2002).

[37] N. Agafonova et al., arXiv:1803.11400.

[38] M. Martini, M. Ericson, G. Chanfray, and J. Marteau, Phys. Rev. C 80, 065501 (2009); M. Martini, M. Ericson, and G. Chanfray, Phys. Rev. D 85, 093012 (2012); 87, 013009 (2013); D. Meloni and M. Martini, Phys. Lett. B 716, 186 (2012); J. Nieves, I. R. Simo, and M. J. Vicente Vacas, Phys. Rev. C 83, 045501 (2011); J. Nieves,
F. Sanchez, I. R. Simo, and M. J. Vicente Vacas, Phys. Rev. D 85, 113008 (2012); O. Lalakulich, K. Gallmeister, and U. Mosel, Phys. Rev. C 86, 014614 (2012); U. Mosel, O. Lalakulich, and K. Gallmeister, Phys. Rev. Lett. 112, 151802 (2014); A. Meucci and C. Giusti, Phys. Rev. D 85, 093002 (2012); G. D. Megias et al., Phys. Rev. D 91, 073004 (2015); P. Coloma and P. Huber, Phys. Rev. Lett. 111, 221802 (2013); J. T. Sobczyk, Phys. Rev. C 86, 015504 (2012).

[39] M. Ericson, M. V. Garzelli, C. Giunti, and M. Martini, Phys. Rev. D 93, 073008 (2016).

[40] M. Antonello et al., arXiv:1503.01520. 